\title{
Recommended System for Educational Literary Tourism Organization Using Information Technologies
}

\author{
Igor Kotsyuba ${ }^{1},{ }^{*}$ Aleksey Shikov ${ }^{2}$, Ruslan Vlasov $^{3}$
}

\author{
${ }^{1}$ ITMO University, Russia \\ ${ }^{2}$ Russian Presidential Academy of National Economy, Russia \\ ${ }^{3}$ Federal State Budgetary Educational Institution of Higher Education «Saint-Petersburg State Institute of \\ Technology», Russia \\ *Email: igor.kotciuba@gmail.com
}

\begin{abstract}
The presented concept of organizing literary tourism describes the development of an information system that allows filling the database with all the necessary information for the implementation of the literary tourism educational goals, taking into account users' needs. One of the development directions of modern educational tourism is specialized for the study of specific academic disciplines. To organize literary tourism at the system level, there is a need to develop a large number of routes, which requires a lot of time and study of huge amounts of information, since it is necessary to study all the works and biography of each of the authors to identify all available places to visit. To optimize this activity, e-tourism tools are actively used. Empirical research methods based on observation and research in the implementation of literary educational tourism using information technology are used. The developed software implements methodological, mathematical, algorithmic software support for the process of organizing literary tourism in St. Petersburg, contributes to a complete study of the literary authors' heritage and the facts of their biography, and also allows the organization of thematic tours in causal relationships on related topics of literary directions. Several requirements have been formulated: support for web application elements, the ability to create a graphical interface, support for object-oriented programming technologies. The designed and implemented information system, due to the ability to automatically extract the needed information, significantly saves time and financial resources of users, which confirms the study's relevance and the need to develop the described information system, which has successfully passed testing and approbation. In the course of surveys of students and teachers, the developed information technology's pedagogical effectiveness was proved for both the student and the teacher. Students could automatically build individual routes and search for works; achieved greater immersion in the educational material due to visualization. The significance of the results obtained lies in students' enthusiasm and motivation for a comprehensive study of literary works, biographies of authors, and historical places in which the storyline is implemented through direct tourist immersion. Prospects for further development imply a complete transition to web technologies to further popularize this information system among a larger user audience of students and teachers.
\end{abstract}

Keywords: Intelligent information system, Information technology, Educational literary tourism, Objectoriented programming.

\section{INTRODUCTION}

Modern education must respond to rapidly changing circumstances both in the organization of the educational process and in the search for new effective methods aimed at the formation of a comprehensively educated person with a culture of thinking, behavior, and erudition. One of the promising forms of organizing the educational process and, at the same time, learning technologies is educational tourism [1]. 


\section{RELATED WORKS}

The use of tourism in integration with the specifics of the educational sphere has a number of advantages, such as:

1. Coverage of both the educational material didactics and the education theory [2].

2. The ability to achieve and consolidate the curriculum goals with the proper level of content "visualization" of several academic disciplines [3].

3. Formation of personally significant qualities both in the field of universal and professional competencies.

4. Introduction to the diversity of cultural, national, and natural potential of a region or state in the patriotic education framework [4].

5. The educational complexity and health-improving work with the use of active recreation methods and sports activities.

6. Expansion of self-realization sphere of a person and his competitiveness. [5]

7. Overcoming both personal and professional crises

8. Adaptation of the population's socially unprotected segments to the socio-cultural environment they carry out their activities [6].

9. The possibility of using a tourist resource in various contexts, for instance: social, informational, cultural, ecological, aesthetic, and contextual combinations [7].

Tourism activities within the educational process meet the main goals of pedagogy, among which are the following:

1. Integration with the teaching and educational activities of the educational institution.

2. Intellectual and moral character of tourism.

3. Relations humanism both in a team and in the field of person formation, taking into account his national, cultural, religious identity.

4. Focus on socially useful needs.

5. Taking into account the student's individual interest and his voluntary participation in tourism activities with a personal choice of types and forms of its organization.

6. A systematic approach to training and education through the scientific and methodological base of its organization.

7. Integration of students and teachers' activities.

8. The organization's planned nature and the continuity of connections in the work of various levels of education.
In addition to pedagogical principles, for the effective implementation of tourism in education, it is crucial to comply with the principles of consistency, multilevel, resource intensity, interdisciplinarity, customer orientation, and interculturality [8].

The implementation of literary tourism has the following advantages in the framework of educational technology:

1. Illustration of reading literary works as a means of enriching theoretical understanding.

2. Demonstration of the writers' connections with the environment of the action of their works.

3. A deeper understanding of the relevance of preserving literary places [9].

4. An empirical assessment of the tourist's awareness of the literary work and the degree of satisfaction with the place [10].

5. Repeated re-reading of literary texts based on personal experience, impressions, and memories of tourist trips.

6. Popularization of literary works as a source material before the start of tourism activities [11].

To organize literary tourism at a systematic level, there is a need to develop a large number of routes, which requires time and study of huge information blocks, since it is necessary to study all the works and biography of each of the authors, to identify all available places to visit. To optimize this activity, e-tourism tools are actively used.

Among the main problems solved with the use of etourism at the present time, one can note:

1. Information updates on popular tourist destinations [12].

2. Helpful travel guides availability [13].

3 . The choice of hotels for multi-day city tourists.

4. Opportunity to tell your stories about visited places and share your experiences [14].

To solve the above-described problems, intelligent information systems can be recommended to analyze a large amount of poorly structured information. Among the methods used in this area, it is worth mentioning the search for Pareto optimality with a heuristic approach with non-dominant sorting [15], multicriteria optimization by popularity, cost and number visited attractions [16], grouping route points using clustering methods to find daily subtasks and taking into account the assessments of users from social networks [12], a genetic algorithm for searching for a neighborhood variable and an algorithm for differential evolution [15], comparing routes with existing typologies of tourism based on motivation [17], with the issuance of 
recommendations based on user behavior on the Internet [18] and others. Nevertheless, the search for new methods of solving the problems of issuing personalized tourist routes does not have a comprehensive solution, and research in this area remains relevant.

\section{MATERIALS AND METHODS}

For centralized information storage in databases, thematic classification of literary movements of the writers of St. Petersburg was carried out:

- classicism;

- sentimentalism;

- romanticism;

- realism;

- modernism (symbolism, acmeism, futurism, imagism);

- socialist realism;

- postmodernism.

To determine the distance between places, store their coordinates in the database in the form of latitude and longitude in a decimal degree measure, and calculate the distance itself using the formula for finding the length of the orthodrome (A.1):

$D=I * \delta$

where $\delta$ - orthodromy angular length, calculated by the formula (A.2):

$\delta=\arccos \left(\sin \varphi_{1} * \sin \varphi_{2}+\cos \varphi_{1} * \cos \varphi_{2} *\right.$ $\left.\cos \left(\lambda_{2}-\lambda_{1}\right)\right)$ $\varphi_{1}$ and $\lambda_{1}-$ latitude and longitude of the location 1;

$\varphi_{2}$ and $\lambda_{2}-$ latitude and longitude of the location 2;

$I$ - arc length $1^{\circ}$ meridian (on Earth $1=111,1 \mathrm{~km}$ ).

Formulas are given without considering polar compression. It should be noted that in this development, the fundamental element is the database. It must be clearly structured, and the connections between its objects must be clearly traced; it must also be flexible, since it can be expanded, and have the proper level of reliability. Relational databases satisfy these requirements.

The application architecture is shown in Fig. 1 using an object-oriented UML component diagram. The system under development uses a client-server three-tier architecture with a dedicated file server since a centralized system implies only one workstation and is too unreliable, which is not suitable for this project, and the file-server system is outdated. Modern development tools allow you to go to the next level at the lowest cost.

This architecture allows to guarantee data integrity and security and allows one to distribute the work of the system between two nodes - a client and a server.

\section{RESULTS}

As a result of the research and based on the specifics of the designed application, the number of requirements was determined:

- $\quad$ ease of development;

- $\quad$ support for elements of web applications;

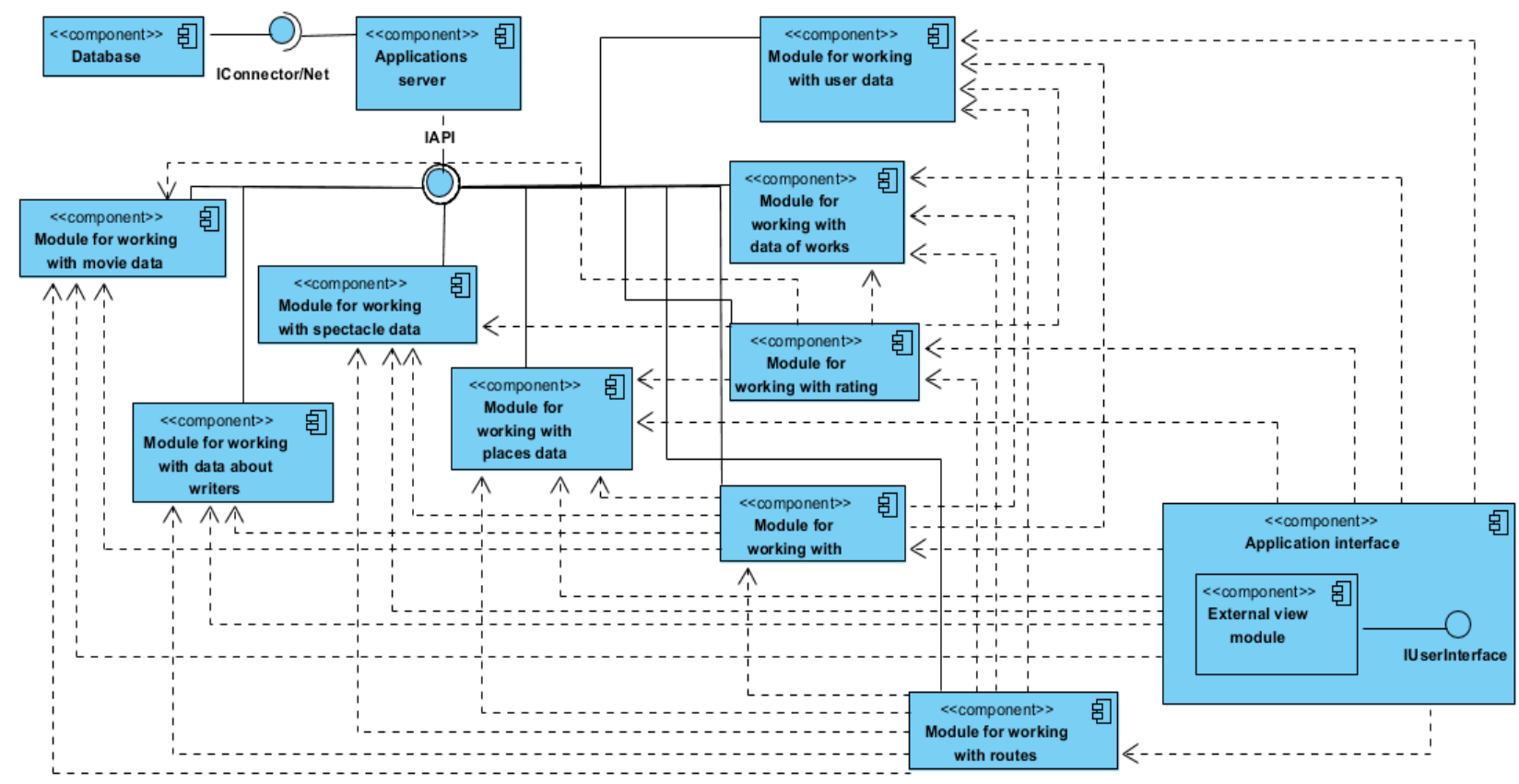

Figure 1 " System architecture". 
- the ability to create a graphical interface;

- support for object-oriented programming.

In particular, these requirements support web application elements, which are met in WinForms and WPF on the .NET platform. They have a special WebBrowser class that allows one to implement an element in an application that possesses all the capabilities and features of the Internet Explorer browser, including support for JavaScript, Dynamic HTML, and plug-ins. Both platforms are designed for creating desktop applications and have a convenient constructor for building UI. However, WPF's functionality is richer, it has XAML support, and the graphics component is based on DirectX. However, the application is a little more complicated; also, WPF is younger than WinForms and lacks a practical guide on the official Microsoft website. Therefore, the development of the client application was implemented in WinForms C \# .NET.

Figures 2-3 show the results of the developed information system approbation on specific examples of literary tourism.

Empirical research results demonstrate estimates of time savings (in minutes), user preferences, and user satisfaction are presented in Figures 4-6.

Prospects for further development imply a complete transition to web technologies to popularize among tourists' large user audience.

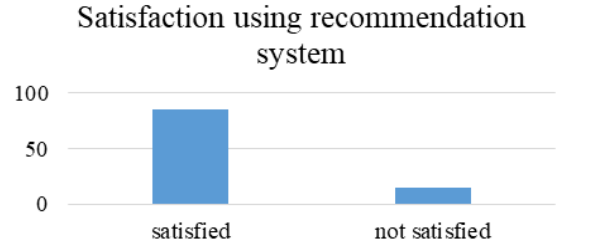

Figure 5 "Assessment of respondents' satisfaction from the software package implementation".

Rating the popularity of tourist object categories

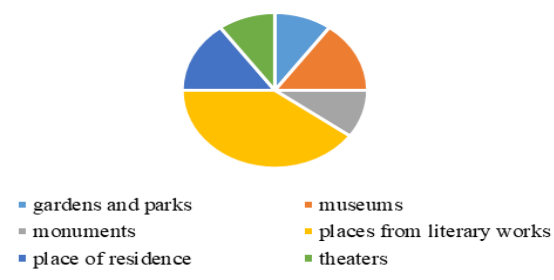

Figure 6 "Rating the popularity of tourist object categories".

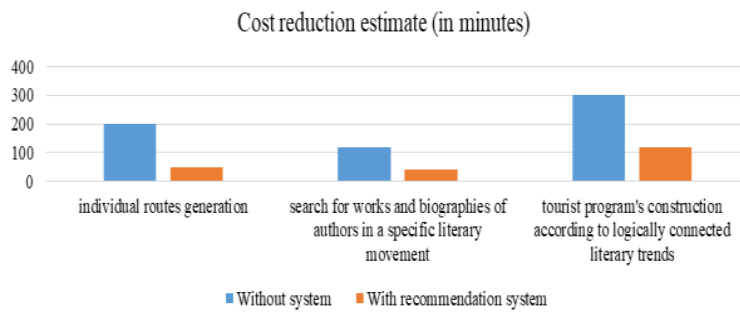

Figure 4 "Assessment of Labor Intensity Reduction".

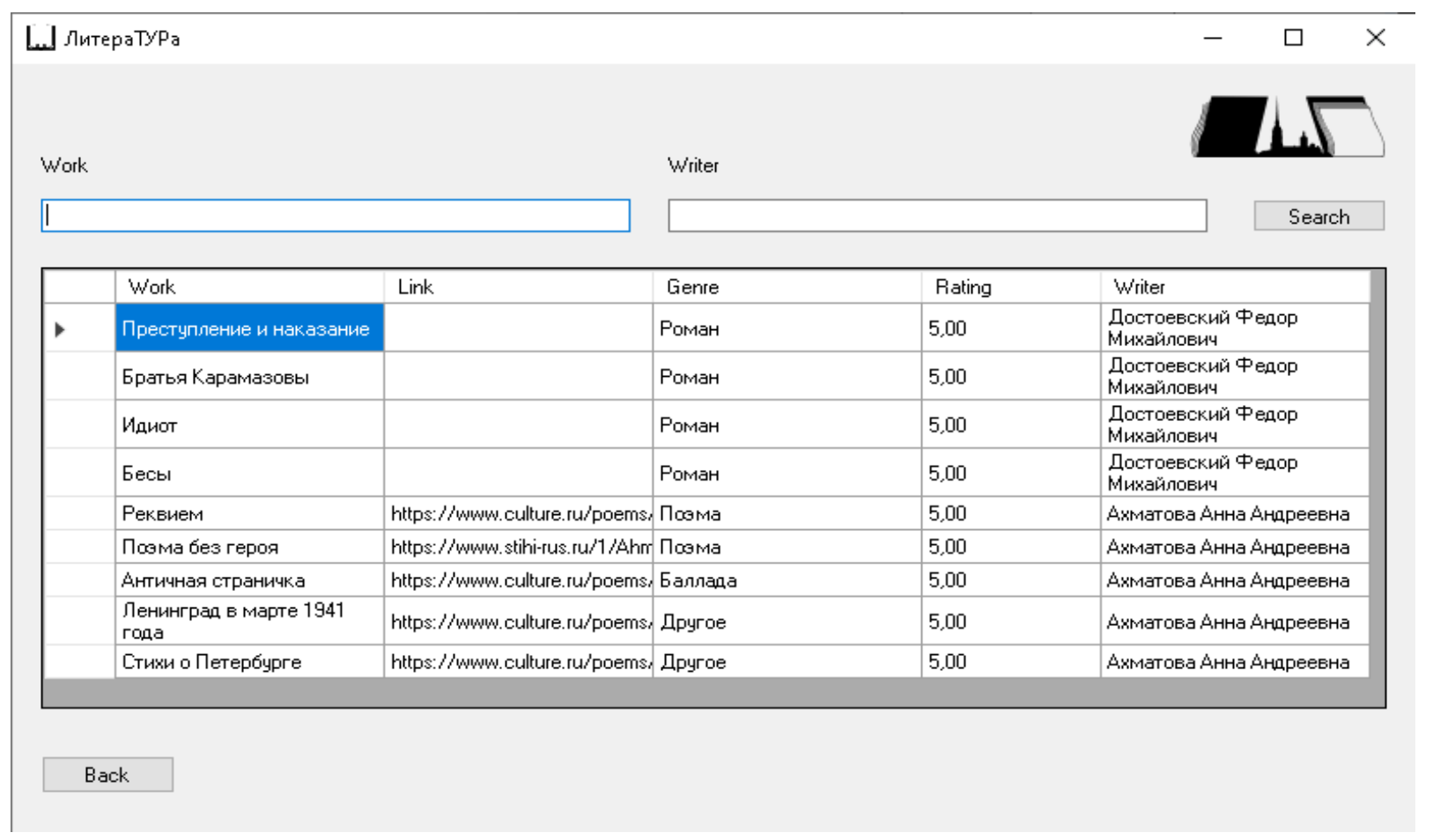

Figure 2 "List of works". 


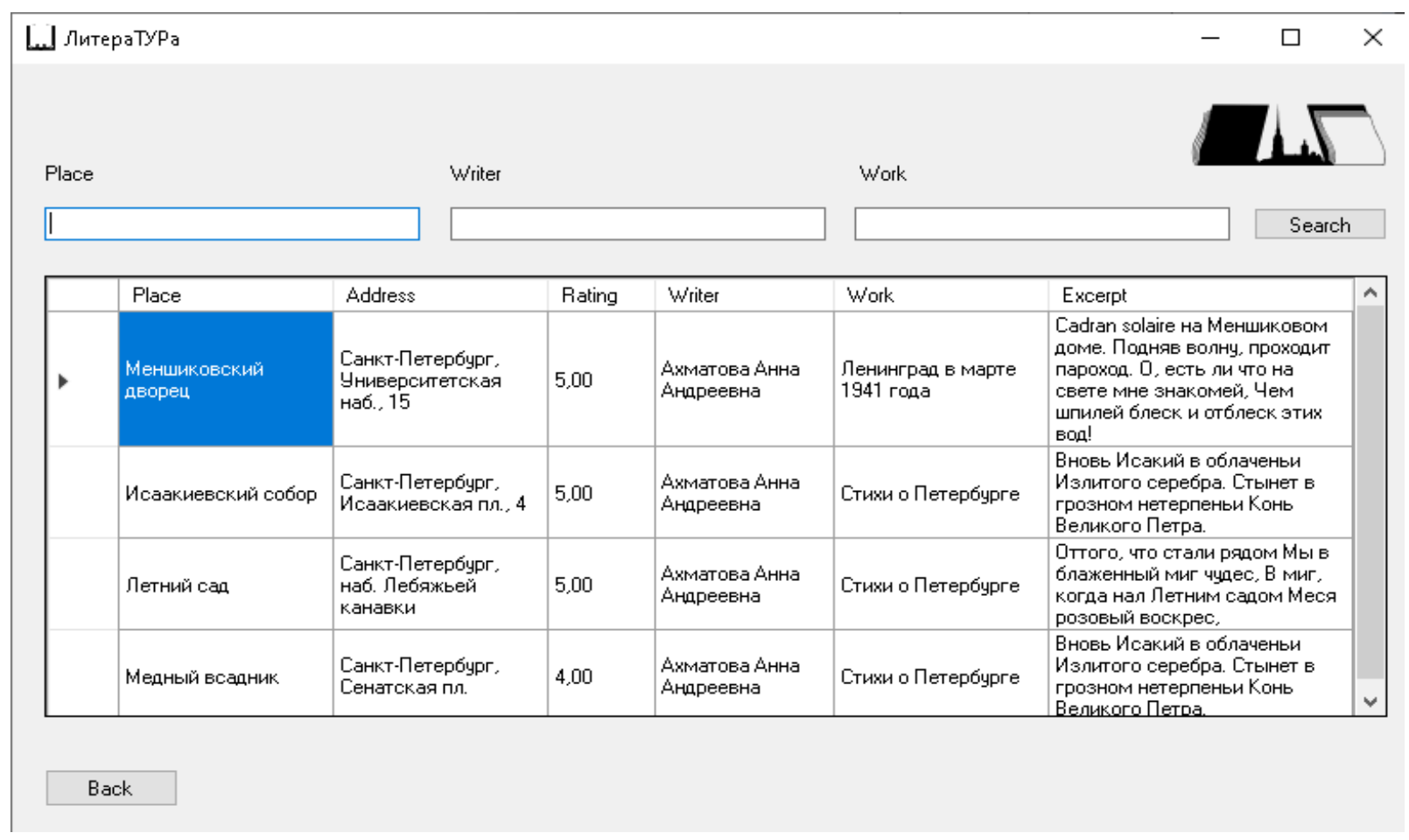

Figure 3 "Window of the places list from works".

Prospects for further development imply a complete transition to web technologies to popularize among tourists' large user audience.

\section{CONCLUSIONS}

The presented information system allows for methodological, mathematical, algorithmic software support for the process of organizing literary tourism in St. Petersburg, and significant savings in time and financial resources confirm the relevance of its creation and use.

The implemented application contributes to a more complete, motivated, and enthusiastic study of the literary heritage of the authors and the facts of their biography, and also allows the organization of thematic tours in causal relationships on related topics of literary trends due to the ability to extract the necessary information from the developed information system automatically.

\section{REFERENCES}

[1] D.O. Golm, G.O. Antiporovich, Educational children and youth tourism as an effective training technology and form of organization of the educational process, Kolpinsky readings on local history and tourism, 2019, pp. 40-45.

[2] A.V. Kolotukha, E.L. Mirgorodskaya, Pedagogical principles of children and youth tourism, Bulletin of the Academy of children and youth tourism and local history 3 (2014) 87-93.

[3] M.N. Pevsner, P.A. Petryakov, A.N. Nikolaeva, From educational management to management of educational tourism: economic and pedagogical aspects, NovSU Bulletin 64 (2011) 59-62.

[4] N.L. Belskaya, Modern approaches to educational tourism management, Scientific result. Business and service technologies 2(4) (2015) 29-38.

[5] N.P. Kushchev, Tourism as an object of sociological analysis in modern Russia, Bulletin of the Lobachevsky University of Nizhny Novgorod. Series: Social Sciences 1(33) (2014) 63-68.

[6] N.V. Molchanova, Tourism as a means of forming tolerance, Bulletin of the Krasnoyarsk state pedagogical University named after V. p. Astafiev 1(35) (2016) 81-84.

[7] A.N. Bardashevich, Tourism as an educational technology, PSE 2(66) (2018) 237-241.

[8] V.D. Ivanov, Educational tourism as a promising direction of youth tourism, Physical culture. Sport. Tourism. Motor recreation 1 (2019) 98-102.

[9] B. Canavan, "Tourism-in-literature: Existential comfort, confrontation and catastrophe in Guy De Maupassant's short stories", Annals of Tourism Research Vol. 78 (2019) 102750. 
[10] D. Herbert, "Literary places, tourism and the heritage experience", Annals of Tourism Research Vol. 28 (2001) 312-33.

[11] N. MacLeod, J. Shelley, A.M. Morrison, "The touring reader: Understanding the bibliophile's experience of literary tourism", Tourism Management Vol. 67 (2018) 388-398.

[12] I. Cenamor, T. de la Rosa, S. Núñez, D. Borrajo, "Planning for tourism routes using social networks", Expert Systems with Applications Vol. 69 (2017) 19.

[13] W. Zheng, H. Ji, C. Lin, W. Wang, B. Yu, "Using a heuristic approach to design personalized urban tourism itineraries with hotel selection", Tourism Management Vol. 76 (2020) 103956.

[14] C. Bassano, S. Barile, P. Piciocchi, J.C. Spohrer, F. Iandolo, R. Fisk, "Storytelling about places: Tourism marketing in the digital age", Cities Vol. 87 (2019) 10-20.

[15] W. Zheng, Z. Liao, "Using a heuristic approach to design personalized tour routes for heterogeneous tourist groups", Tourism Management Vol. 72 (2019) 313-325.

[16] M.A. Uwaisy, Z.K.A. Baizal, M.Y. Reditya, "Recommendation of Scheduling Tourism Routes using Tabu Search Method (Case Study Bandung)", Procedia Computer Science Vol. 157 (2019) 150159.

[17] S. Ma, A.P. Kirilenko, S. Stepchenkova, "Special interest tourism is not so special after all: Big data evidence from the 2017 Great American Solar Eclipse", Tourism Management Vol. 77 (2020) 104021.

[18] M. Sumardi, R. Wongso, F.A. Luwinda, "TripBuddy" Travel Planner with Recommendation based on User's Browsing Behaviour", Procedia Computer Science Vol. 116 (2017) 326-333. 